\title{
A Randomized Double-blind, Sham-stimulation Control Pilot Study of the Effect of an Electromagnetic Field Generator on Healthy Subjects
}

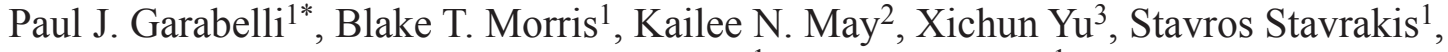 \\ Benjamin J. Scherlag ${ }^{1}$ and Sunny S. Po ${ }^{1}$ \\ ${ }^{1}$ University of Oklahoma Health Science Center, Cardiovascular Diseases Section, USA; ${ }^{2}$ University of Oklahoma College of Medicine, \\ USA; ${ }^{3}$ University of Oklahoma Health Science Center, Diabetes and Endocrinology Section, USA
}

\begin{abstract}
Background and objective: Electromagnetic fields have been evaluated in multiple environments as a source of incidental exposure as well as a therapeutic treatment modality. At low levels and in specific therapies, electromagnetic fields have been found to be safe and may have positive effects on human physiology that improve disease course or alter the severity of some ailments. We conducted a study to investigate whether a commercial Federal Drug Administration-approved electromagnetic field generator device used for stress reduction and relaxation has the capability to impart a measurable impact on the cardiac autonomic nervous system.
\end{abstract}

Methods: This study was designed as a randomized double-blind, sham-stimulation controlled study in healthy subjects. The stimulation or treatment was the application of parasympathetic-targeted electromagnetic fields through the Federal Drug Administration-approved Resonator ${ }^{\circ}$ device for $60 \mathrm{~m}$. The primary measurements of treatment effect were heart rate variability (measured at baseline and every $15 \mathrm{~m}$ ) and salivary cortisol (pre- and postapplication). The active treatment consisted of two sessions, $60 \mathrm{~m}$ in duration. The electromagnetic field algorithm applied was A160 (3.1 $\times 10^{-8}$ to $3.2 \times 10^{-8}$ Gauss, frequency range of $\left.0.857-0.859 \mathrm{~Hz}\right)$. All participants received both a sham and an active treatment session, the order of which was randomized.

Results: A total of 28 patients completed both sessions. The measured difference in cortisol between the groups was not statistically significant $(p=0.66)$. There was a trend towards decreased cortisol levels over time in both groups ( $p=0.09$ for time trend). Measured heart rate variability showed a nonsignificant reduction in low frequency to high frequency ratio (low frequency/high frequency) at $60 \mathrm{~m}$.

Conclusions: While the results were nonsignificant, the trend towards a reduction in low frequency/high frequency is suggestive of a delayed electromagnetic field effect. This study is hypothesis-generating since additional research is needed to either adjust the electromagnetic field treatment dose/duration during sessions or delay the final data collection of heart rate variability and salivary cortisol until hours after the active treatment.

Keywords: Electromagnetic fields; Heart rate variability; Autonomic nervous system. Received: November 02, 2017; Revised: February 28, 2018; Accepted: March 13, 2018

${ }^{*}$ Correspondence to: Paul Garabelli, 5224 E. I-240 Service Road, Suite 100, Oklahoma City, OK 73135, USA. Fax: 405-972-7568; E-mail: pgarabelli@gmail.com How to cite this article: Garabelli PJ, Morris BT, May KN, Yu X, Stavrakis S, Scherlag BJ, Po SS. A Randomized Double-blind, Sham-stimulation Control Pilot Study of the Effect of an Electromagnetic Field Generator on Healthy Subjects. Exploratory Research and Hypothesis in Medicine 2018;3(2):28-32. doi: 10.14218/ ERHM.2017.00031.
Introduction

Electromagnetic field stimulation is known to affect the central nervous system. Previously, electromagnetic fields were studied for the treatment of depression and other neurologic disorders, including Parkinson's disease and multiple sclerosis. ${ }^{1-4}$ The proposed mechanism through which very weak, low frequency (or picoTesla) electromagnetic fields cause biologic amplification has been described in a hypothetical mathematical construct by Saxena et al..$^{5}$ The physics of electromagnetic field biologic amplifications 


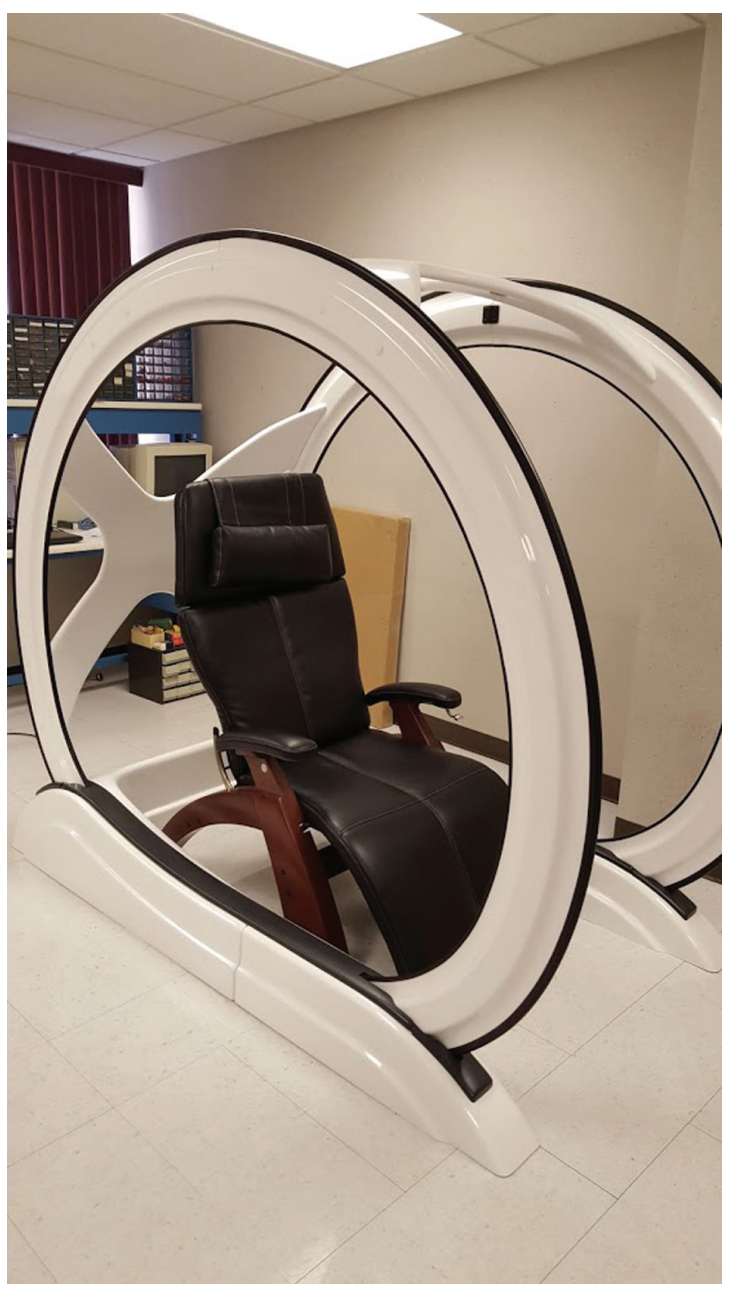

Fig. 1. The Resonator ${ }^{\circledR}$ device. The simplified Helmholtz coil system is 7 feet in diameter with 3.5 feet of separation. The system is designed to create a homogenous, isotropic magnetic field from 1 to 99 pT with a frequency range of $0.01 \mathrm{~Hz}$ to $50 \mathrm{~Hz}$.

are complex; however, recent in vivo controlled studies have offered empiric support for this hypothesis.

Our group recently showed that low-level electromagnetic field application in a dog model could suppress atrial fibrillation, the most common clinical arrhythmia in practice, through modulation of the dominant frequencies of the cardiac autonomic nervous system. ${ }^{6}$ Indeed, the effect of electromagnetic fields on atrial fibrillation is thought to be secondary to modulation of the cardiac autonomic nervous system, which plays a role in initiation and maintenance of ventricular as well as atrial arrhythmias. These results were intriguing as they suggested the modality as a potential noninvasive treatment option for atrial fibrillation through modulation of the cardiac autonomic nervous system. Considering the epidemic that atrial fibrillation is becoming and the inadequacy of current treatments for it, additional treatment options for patients suffering from this rhythm problem are paramount. ${ }^{7}$

We studied the effect of a commercial second generator electromagnetic field device called the Resonator ${ }^{\circledR}$ (Magneceutical Health, Clearwater, FL, USA) on the cardiac autonomic nervous system. The Resonator ${ }^{\circledR}$ (Fig. 1) is Federal Drug Administrationapproved and is currently used as a therapeutic device to enhance relaxation and well-being. However, to our knowledge, there is no published scientific data on the Resonator ${ }^{\circledR}$ and its effect on the cardiac autonomic nervous system.

\section{Methods}

This study was designed as a randomized double-blind, shamstimulation controlled study and was approved by our institutional review board (IRB \#6134, approval date 12/18/2015). Informed consent, research methods and data collection were followed in strict accordance to the rules set forth by the Institutional Review Board of the University of Oklahoma. No conflicts of interest were reported by any investigators.

The stimulation or treatment consisted of the application of electromagnetic fields through the Federal Drug Administrationapproved Resonator ${ }^{\circledR}$. The primary measurements of treatment effect were heart rate variability and salivary cortisol values. Healthy subjects were recruited from the faculty and staff within the department of cardiology at the University of Oklahoma Health Sciences Center. Informed consent was obtained from all subjects. Participants were excluded if they answered yes to any of the following queries: known left ventricular dysfunction (left ventricular ejection fraction $<40 \%$ ), significant valvular disorder (i.e. prosthetic valve or hemodynamically relevant valvular diseases), recent ( $<6$ months) stroke or myocardial infarction, severe heart failure (NYHA IV), left atrial dilatation $(>55 \mathrm{~mm})$, recurrent vasovagal syncopal episodes, unilateral or bilateral vagotomy, pregnancy or breastfeeding, intracardiac devices (i.e. defibrillators and pacemakers), or recurrent oral bleeding.

Included subjects were then asked to avoid eating a major meal for $60 \mathrm{~m}$ prior to the study and to refrain from alcohol use for 12 $\mathrm{h}$ prior to the appointment. They were also asked not to consume anything with high acidity or high sugar content within $30 \mathrm{~m}$ of the study. All participants rinsed their mouth with water upon check-in and study personnel verified adherence to the rules.

\section{Resonator $^{\circledR}$}

The Resonator ${ }^{\circledR}$ is controlled by a computer connected to a magnetic driver. It contains a signal generator with an attenuator circuit prepared for a Helmholtz coil to produce the magnetic field. The simplified Helmholtz coil system is 7 feet in diameter with 3.5 feet of separation, as shown in Figure 1. The system is designed to create a homogenous, isotropic magnetic field from 1 to $99 \mathrm{pT}$ with a frequency range of $0.01 \mathrm{~Hz}$ to $50 \mathrm{~Hz}$.

During the sessions, participants wore head phones to reduce effects of any outside noise. Pagers, cell phones and other electronic equipment were placed in airplane mode and left on a table across the room for the duration of the treatment. The participant sat in a zero gravity chair (Fig. 1) which was set at 40 degrees and remained fixed during the sessions. Likewise, the chair remained inside the Helmholtz coil for the duration of the study. Since each participant was required to stay awake during the sessions, they were asked to read the same book "And Then There Were None" by Agatha Christie. Study personnel remained in the room to ensure adherence. The active treatment session was the A160 algorithm. This was a narrow range but represented the variable magnetic field that was described by the manufacturer as the standard parasympathetic setting. It was a range of $3.1 \times 10^{-8}$ to $3.2 \times 10^{-8}$ Gauss, with a frequency range of $0.857-0.859 \mathrm{~Hz}$.

All participants received both a sham and an active treatment 
Table 1. Demographics of the study population

\begin{tabular}{ll}
\hline \multicolumn{2}{c}{ Demographic feature } \\
\hline Age in years, mean (SD) & $43( \pm 13)$ \\
Male sex & $54 \%$ \\
\hline
\end{tabular}

Note: The data describe the demographics of the 28 participants. Besides each subject's required 'no' answer to the exclusion criteria, no additional health information was collected on each subject.

session, each of which was $60 \mathrm{~m}$ in duration. The order of the sessions (either sham first or treatment first) was randomly assigned to each participant. After completion of the first session, the subject "crossed-over" into the opposite treatment arm. The second session (the opposite of the first session) was performed within 1 week of the first session.

Participants and researchers performing the study were blinded to which session was treatment or sham. Before unblinding, the two therapy session types were preloaded into the Magnesphere computer remotely via a person not involved with any aspect of the study. These were labeled "Program A" and "Program B". Neither the participants nor the researchers knew whether "Program A" was the active session or sham session. At completion of the study, the researchers were unblinded.

\section{Heart variability measurement}

Heart rate variability is derived through a measurement of beat-tobeat variability in normal sinus rhythm and can be quantified by several methods based on changes in underlying parasympathetic and sympathetic input represented in the measured data. ${ }^{8}$ Data for heart rate variability analysis were recorded through electrocardiogram electrodes placed on both arms and legs of each participant and which remained on the participant for the duration of the study. The PC electrocardiogram V2.12 SEMIP V1.61 ${ }^{\odot} 2012$ software was used. Each heart rate variability reading was $5 \mathrm{~m}$ in duration. A baseline measurement was taken after the subject was comfortable in the chair, but before initiation of treatment. Additional sampling was performed during the session (sham or active) from time

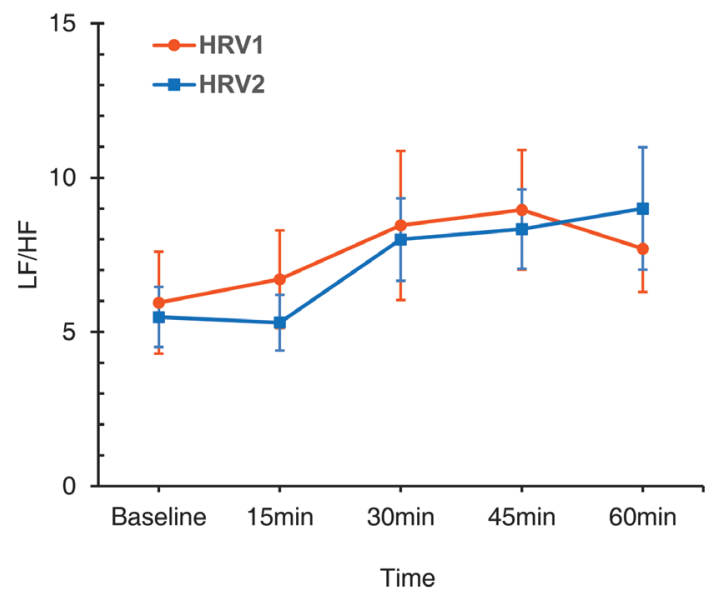

Fig. 2. Heart rate variability. Heart rate variability was analyzed using the low frequency/high frequency ratio. Heart rate variability-1 (circles, black) indicate the active electromagnetic field session and heart rate variability-2 (squares, red) indicate the sham session. Vertical bars represent standard error of the mean.
Table 2. Salivary cortisol

\begin{tabular}{llll}
\hline Time & Sequence & Estimate & Standard error \\
\hline 0 & sham & 0.2818 & 0.0603 \\
0 & treatment & 0.3055 & 0.0603 \\
1 & sham & 0.1954 & 0.0613 \\
1 & treatment & 0.2364 & 0.0603 \\
\hline
\end{tabular}

marks $10-15 \mathrm{~m}, 25-30 \mathrm{~m}, 40-45 \mathrm{~m}$, and 55-60 m. Information collected included heart rate (beats per m), SDNN, RMSSD (ms), PNN50 (\%), total power (ms*ms), low frequency (ms*ms), high frequency $\left(\mathrm{ms}^{*} \mathrm{~ms}\right)$, low frequency norm, high frequency norm, and low frequency/high frequency.

\section{Salivary cortisol collection}

The sessions were scheduled at $7 \mathrm{am}, 8 \mathrm{am}$ or 9am in an attempt to capture peak cortisol in patients. A salivary collection system with oral swabs (Salimetrics, LLC, State College, PA, USA) was used to collect saliva samples in the baseline state and immediately following each $60-\mathrm{m}$ session. The manufacturer's instructions were followed for saliva collection. After collection, the swab was placed into a test tube for cryostorage. All saliva samples were analyzed together using the ER HS Salivary Cortisol Kit, again following the manufacturer's instructions.

\section{Statistical analysis}

This was a feasibility pilot study. The primary outcome of this study was defined as a change in cardiac autonomic nervous system as measured by heart rate variability and salivary cortisol levels. The null hypothesis was that there would be no difference between salivary cortisol levels and heart rate variability after treatment. Markers of the cardiac autonomic nervous system were compared between groups using the statistical method for analysis of variance. Statistical significance was declared at $p<0.05$.

Sample size was estimated based on a power calculation. The change in the low frequency component, which is a reflection of sympathetic tone, was used to calculate the sample size. Assuming a $40 \%$ decrease in low frequency with the Resonator ${ }^{\circledR}$ and a coefficient of variation of 0.8 , a sample size of 32 subjects was estimated to achieve $80 \%$ power in a $2 \times 2$ cross-over design at a significance level of 0.05 .

All statistical analyses were performed using SAS version 9.3 (SAS Institute Inc., Cary, NC, USA).

Results

A total of 28 patients completed both sessions. The average age of the participants was $44 \pm 13$ years-old and $54 \%$ were male, as shown in Table 1. Heart rate variability was analyzed using low frequency/high frequency ratio, as shown in Figure 2, where heart rate variability- 1 was the active electromagnetic field session and heart rate variability-2 was the sham session. There was a nonsignificant reduction in low frequency/high frequency ratio at the final reading within the study, suggestive of increased parasympathetic activity.

Cortisol samples were thawed and analyzed together to reduce 
error. A total of six cortisol sessions had to be excluded secondary to inadequate saliva for analysis. The measured difference in the cortisol $(\mu \mathrm{g} / \mathrm{dL})$ between the groups was not statistically significant $(p=0.66)$, as shown in Table 2. However, there was a trend towards decreased cortisol levels over time in both groups ( $p=$ 0.09 for time trend).

\section{Discussion}

Electromagnetic fields have been well studied and the modality is already being used to treat depression, seizures, Parkinson's disease, and multiple sclerosis. ${ }^{1-4}$ As the population ages and as chronic diseases increase in prevalence, finding noninvasive treatment options is critical. Understanding that appropriately targeted electromagnetic fields can impact the cardiac autonomic nervous system and potentially mitigate symptoms from some chronic diseases, such as atrial fibrillation has relevance for treatment considerations; however, there is still debate in the literature on the role of electromagnetic fields, some of which prompted our study. For example, Lewczuk et $a l .{ }^{9}$ provided several animal and human studies which have shown contradictory information on how electromagnetic fields affect circadian rhythm and cortisol secretion. Similarly, since the electromagnetic field effect is dependent on intensity and exposure time for specific cellular targets, the potential exists to cause unintended changes in other cellular sites. As the scientific field of low powered electromagnetic fields grows, data on appropriate treatments for different biologic systems will continue to accrue.

We designed this pilot study to demonstrate the effect of one 60-m parasympathetic-focused electromagnetic field session using the Resonator ${ }^{\circledR}$ on the cardiac autonomic nervous system in healthy subjects. Neither the heart rate variability (as measured by low frequency/high frequency) nor the salivary cortisol levels were statistically significant. However, both measures showed trends towards significance. The trend towards decreased cortisol levels over time in both groups suggested that a $60-\mathrm{m}$ session in a zero gravity chair (whether sham or active electromagnetic field) was stress reducing.

The reduction in the low frequency/high frequency ratio at the $60-\mathrm{m}$ mark in only the active electromagnetic field session was suggestive of a parasympathetic effect on subjects, possibly due to the Resonator ${ }^{\circledR}$. Yu et al. ${ }^{6}$ reported a "pharmacologic" or delayed effect in an electromagnetic field dog model studying atrial fibrillation. The electromagnetic field was delivered through a Helmholz coil and suppressed the atrial fibrillation during the electromagnetic field therapy in addition to hours following therapy. ${ }^{6}$ This theory of a delayed effect also mirrors the trend seen in our data, namely the reduction in low frequency/high frequency at $60 \mathrm{~m}$. While our data was not statistically significant, it is possible that a statistically significant reduction of low frequency/high frequency would be seen at 60 or $120 \mathrm{~m}$ following therapy or after multiple sessions.

Importantly, there are several limitations to our study. First, the salivary cortisol measurement was not adequate in $16 \%$ of the samples (or 6 total sessions). Repeating the study with a different measurement technique utilizing passive drool after a period of chewing sugarless gum may improve data collection. ${ }^{10}$ Furthermore, there may have not been enough time within the treatment sessions to see a difference. A recent study evaluating occupational exposure to electromagnetic fields in dental practice showed significant reductions in serum cortisol levels in dentists and dental students exposed to electromagnetic radiation using routine dental equipment over a period of roughly $4 \mathrm{~h} .{ }^{11}$ Repeating our study with a longer treatment session, measuring cortisol $>1 \mathrm{~h}$ after the treat- ment ends, or use of a serum rather than a salivary measurement may yield different results.

Secondly, according to the manufacturer, the Resonator ${ }^{\circledR}$ was not designed to be a single treatment. During commercial use, the sessions are performed in series, such that each treatment session builds upon the previous session. Repeating the study with the same population but with 5-6 sessions over 2 weeks may show a greater response. Further individual subject adjustments to the electromagnetic field program based on each subject's response may lead to more statistically significant results.

Third, the patient population studied may not have had enough stress or chronic illnesses. As previously studied, the cardiac autonomic nervous system has been shown to play a role in inflammatory pathways and inflammatory cells. ${ }^{12}$ Repeating the study with a population with known stress or chronic illness, such as diabetes, atrial fibrillation or rheumatoid arthritis, may show a statistically significant difference between treatment groups in just one session.

Finally, the study was underpowered to detect a difference. While 32 subjects were needed based on initial power calculations, only 28 subjects completed both sessions. Recruitment of healthy cardiology department subjects was more difficult than expected, and the primary investigator was moving from the University to a different position. Based on this information, a discussion amongst the researchers was held. Considering that this was a feasibility pilot study, a decision was made to stop recruitment and analyze the results with only 28 subjects.

\section{Conclusions and future directions}

While our pilot study demonstrated no statistical difference in salivary cortisol or heart rate variability after a $60-\mathrm{m}$ parasympathetic-focused electromagnetic field session using the Resonator ${ }^{\circledR}$ in healthy subjects, the trend in heart rate variability response was encouraging. Using this data as a launching point, additional studies can be designed to better tailor electromagnetic fields from the Resonator ${ }^{\circledR}$ to the population being studied. For example, patients with chronic inflammatory diseases, such as diabetes, will potentially have higher levels of cortisol and their inclusion may therefore show more of a response to therapy.

Note: The measured difference in the cortisol $(\mu \mathrm{g} / \mathrm{dL})$ between the groups was not statistically significant $(p=0.66)$. Time 0 represents presession (sham or treatment) and Time 1 represents postsession (sham or treatment).

\section{Acknowledgments}

The authors would like to especially thank Kathy Drennan, Karrie Meeks and Zachary Nuss for their administrative and research support during the development, implementation and data organization performed for this study.

\section{Conflict of interest}

The authors have no conflict of interests related to this publication.

\section{Author contributions}

Experiment design (PJG, XY, SS, BJS, SSP), oversight (PJG, BJS, 
Explor Res Hypothesis Med

SSP), manuscript writing (PJG, BTM, KNM, SSP), data collection (BTM, KNM), data analysis (KNM, XY, SS).

\section{References}

[1] Sandyk R. Magnetic fields in the therapy of parkinsonism. Int J Neurosci 1992;66(3-4):209-235.

[2] Sandyk R. Successful treatment of multiple sclerosis with magnetic fields. Int J Neurosci 1992;66(3-4):237-250.

[3] Anninos PA, Tsagas N, Sandyk R, Derpapas K. Magnetic stimulation in the treatment of partial seizures. Int J Neurosci 1991;60(3-4):141-171.

[4] Sobis J, Jarzab M, Hese RT, Sieron A, Zyss T, Gorczyca P, et al. Therapeutic efficacy assessment of weak variable magnetic fields with low value of induction in patients with drug-resistant depression. J Affect Disord 2010;123(1-3):321-326. doi:10.1016/j.jad.2009.09.016.

[5] Saxena A, Jacobson J, Yamanashi W, Scherlag B, Lamberth J, Saxena B. A hypothetical mathematical construct explaining the mechanism of biological amplification in an experimental model utilizing picoTesla (PT) electromagnetic fields. Med Hypotheses 2003;60(6):821-839. doi:10.1016/S0306-9877(03)00011-2.

[6] Yu L, Dyer JW, Scherlag BJ, Stavrakis S, Sha Y, Sheng X, et al. The use of
Garabelli PJ. et al: EMF generator effect on healthy subjects

low-level electromagnetic fields to suppress atrial fibrillation. Heart Rhythm 2015;12(4):809-817. doi:10.1016/j.hrthm.2014.12.022.

[7] Weerasooriya R, Khairy P, Litalien J, Macle L, Hocini M, Sacher F, et al. Catheter ablation for atrial fibrillation: are results maintained at 5 years of follow-up? J Am Coll Cardiol 2011;57(2):160-166. doi:10.1016/j.jacc.2010.05.061.

[8] Malik M, Camm AJ. Components of heart rate variability-what they really mean and what we really measure. Am J Cardiol 1993;72(11):821-822. doi:10.1016/0002-9149(93)91070-X.

[9] Lewczuk B, Redlarski G, Zak A, Ziolkowska N, Przybylska-Gornowicz $B$, Krawczuk M. Influence of electric, magnetic, and electromagnetic fields on the circadian system: current stage of knowledge. Biomed Res Int 2014;2014:169459. doi:10.1155/2014/169459.

[10] Dabbs JM Jr. Salivary testosterone measurements: collecting, storing, and mailing saliva samples. Physiol Behav 1991;49(4):815-817. doi:10.1016/0031-9384(91)90323-G.

[11] Mortazavi SM, Vazife-Doost S, Yaghooti M, Mehdizadeh S, Rajaie-Far A. Occupational exposure of dentists to electromagnetic fields produced by magnetostrictive cavitrons alters the serum cortisol level. $J$ Nat Sci Biol Med 2012;3(1):60-64. doi:10.4103/0976-9668.95958.

[12] Hasko G. Receptor-mediated interaction between the sympathetic nervous system and immune system in inflammation. Neurochem Res 2001;26(8-9):1039-1044. doi:10.1023/A:1012305122327. 